\title{
Numerical study for failure behavior of rock masses including complex free-surfaces using a particle method
}

\author{
Junichi TAKEKAWA ${ }^{1}$, Hitoshi MIKADA ${ }^{1}$ and Tada-nori GOTO ${ }^{1}$ \\ ${ }^{1}$ Dept. of Civil and Earth Res. Eng., Kyoto University
}

\begin{abstract}
Failure of rock mass including complex free surfaces is of importance in many engineering and scientific fields. This paper applied an advanced discretization approach to simulate quasi-static failure of rock mass within a Hamiltonian particle method (HPM) framework. In HPM, a free surface is introduced in a simple way, just by removing or ignoring outer particles. This potential can be developed to discretize numerical models including complex free surfaces without the increment of time for pre-processing. In the present study, we developed the numerical simulator based on HPM with a staggered particle technique for simulating brittle failure and AE activities in rock mass with incorporating the elasto-plastic damage model. We, first, conducted uni-axial compressive tests for validating the effectiveness of our approach. Next, we adopted rectangular and circular disc specimens with a hole as complex free surface models. Our numerical results had good agreement with those from laboratory experiments. This suggests that HPM would be a method to simulate failure behavior of rock mass without time-consuming pre-processing.
\end{abstract}

\section{INTRODUCTION}

It is important to understand failure behavior of rock mass in many scientific and engineering fields. In the past decades, many numerical approaches have been developed and applied to simulate the failure behavior of rock mass ${ }^{1), 2 \text {. }}$. As a numerical method, finite difference methods (FDM) or finite element methods (FEM) have often been used. On the other hand, other numerical methods have adopted as the numerical simulator. Potyondy and Cundall ${ }^{3)}$ applied distinct element method (DEM) for analyses of failure of rock. Feng and co-workers 4) developed an elasto-plastic cellular automaton method for simulation of the rock micro-fracturing process under uni-axial compression. Smoothed particle hydrodynamics ( $\mathrm{SPH}$ ) method was also applied to failure simulations of rock mass ${ }^{5}$. Since the $\mathrm{SPH}$ is one of the particle methods, it has several advantages over other traditional continuum methods.

A Hamiltonian particle method (HPM) is also a particle method, and was recently developed for solving incompressible fluid flows 6), and non-linear elastodynamics 7). Since the HPM is based on the mesh-free approach, complex free surfaces and large deformations can be dealt with in a simple manner. These advantages of the HPM would be developed to the analysis of the brittle failure of rock masses including complex free surfaces. Although the HPM has been applied to the estimation of the strong ground motion induced by an earthquake ${ }^{8)}$, and the rock physical study for cracked media ${ }^{9)}$, the applicability of the HPM to the brittle failure analysis has not been revealed yet.

In the present study, we apply the HPM to the simulation of the brittle failure behavior and $\mathrm{AE}$ activities of rock mass including complex free surfaces. We, first, conduct uni-axial compressive test. Next, the failure of a circular disc with an eccentric hole is simulated for validating the effectiveness of the HPM.

\section{METHOD}

In this section, we explain the basic theory of HPM and the staggered particle strategy ${ }^{10}$. Stress and displacement components are defined at main and sub-particles, respectively. In the HPM, the deformation gradient tensor is calculated by minimizing the error function $\mathrm{e}_{\mathrm{i}}$ as follows.

$e_{\mathrm{i}}=\sum_{\mathrm{j}}\left|\boldsymbol{F}_{\mathrm{i}} \boldsymbol{r}_{\mathrm{ij}}^{0}-\boldsymbol{r}_{\mathrm{ij}}\right|$

where $\mathbf{F}$ is the deformation gradient tensor, $\boldsymbol{r}_{\mathrm{ij}}^{0}$ and $\boldsymbol{r}_{\mathrm{ij}}$ are the initial and current position of sub-particle j relative to main-particle $i$, respectively. Subscripts $\mathrm{i}$ and $\mathrm{j}$ indicate main- and sub-particle, respectively. The summation in Eq.(1) is applied to the surrounding sub-particles inside the influence domain of main-particle i. Minimizing the error function derives the following equation for 
calculating the deformation gradient tensor.

$\boldsymbol{F}_{\mathrm{i}}=\sum_{\mathrm{j}} \boldsymbol{r}_{\mathrm{ij}} \otimes \boldsymbol{r}_{\mathrm{ij}}^{0} A_{\mathrm{i}}^{-1}$

$\boldsymbol{A}_{\mathrm{i}}=\sum_{\mathrm{j}} \boldsymbol{r}_{\mathrm{ij}}^{0} \otimes r_{\mathrm{ij}}^{0}$

$a \otimes b$ means tensor product of vector $\mathbf{a}$ and $\mathbf{b}$. The strain tensor, stress tensor and the total elastic strain energy can be calculated using the deformation gradient tensor.

$\boldsymbol{E}_{\mathrm{i}}=\frac{1}{2}\left(\boldsymbol{F}_{\mathrm{i}}^{\mathrm{T}} \boldsymbol{F}_{\mathrm{i}}-\boldsymbol{I}\right)$

$\boldsymbol{S}_{\mathrm{i}}=2 \mu \boldsymbol{E}_{\mathrm{i}}+\lambda \operatorname{tr}\left(\boldsymbol{E}_{\mathrm{i}}\right) \boldsymbol{I}$

$V=\sum_{\mathrm{i}} \frac{1}{2}\left(\boldsymbol{S}_{\mathrm{i}}: \boldsymbol{E}_{\mathrm{i}} \Delta \mathrm{B}_{\mathrm{i}}\right)$

where $\mathbf{E}, \mathbf{S}$ and $\mathrm{V}$ are the Green-Lagrangian strain tensor, second Piola-Kirchhoff stress tensor and the total elastic strain energy, respectively. $\Delta B_{i}$ is the volume of main-particle i. Using Hamiltonian's equation, we can derive the equation of motion for each sub-particle $\mathrm{j}$.

$$
\begin{aligned}
\Delta \mathrm{m}_{\mathrm{j}} \frac{\partial v_{\mathrm{j}}}{\partial \mathrm{t}} & =\frac{\partial V}{\partial \boldsymbol{r}_{\mathrm{j}}} \\
& =\sum_{\mathrm{i}}\left(\boldsymbol{F}_{\mathrm{i}} \boldsymbol{S}_{\mathrm{i}} \boldsymbol{A}_{\mathrm{i}}^{-1} \boldsymbol{r}_{\mathrm{ji}}^{0} \Delta \mathrm{B}_{\mathrm{i}}\right)
\end{aligned}
$$

where $\Delta \mathrm{m}_{\mathrm{j}}$ is the mass of sub-particle $\mathrm{j}$. We apply a simplectic scheme to Eq.(7) for solving the time marching with sufficient accuracy. Details are shown in a reference ${ }^{10)}$.

\section{NUMERICAL RESULTS}

\section{(1) Uni-axial compressive test}

Figure 1 shows the configuration of the uni-axial compressive test. A rectangular specimen is compressed by a constant strain.

Figure 2 shows the damage evolution of the

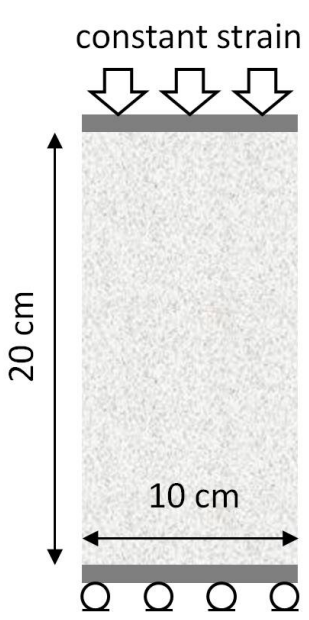

Figure 1: Model configuration of the uni-axial compressive test. The specimen is compressed under a constant strain.

specimen. The microscopic failures interact, propagate, and coalesce each other. Finally, the macroscopic failure plane is formed obliquely.

Figure 3 shows the stress-strain and cumulative $\mathrm{AE}$ counts curves. The stress increases almost linearly in the early loading stage. Around peak strength, the non-linear behavior of the stress-strain curve can be observed. The number of AE activities increases gradually in the early loading stage. Around peak strength, AE counts increase rapidly. Our numerical result has good agreement with that from laboratory experiments in terms of the microscopic and macroscopic behavior.

\section{(2) Failure of a disc with an eccentric hole}

Figure 4 shows the description of a circular disc with an eccentric hole. The eccentricity and radius of the hole are parameters in this section. We test two sets of parameters, (1) e $=32 \mathrm{~mm}, \mathrm{a}=28 \mathrm{~mm}$,

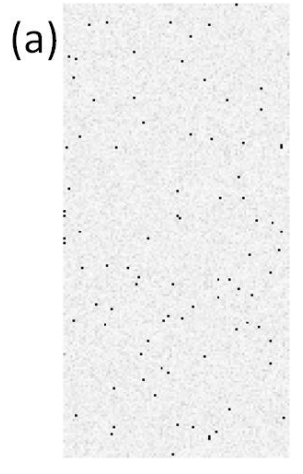

$75 \%$ peak (b)

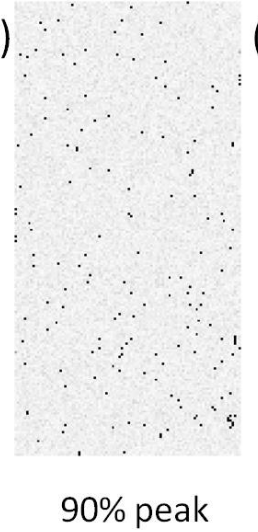

(c)

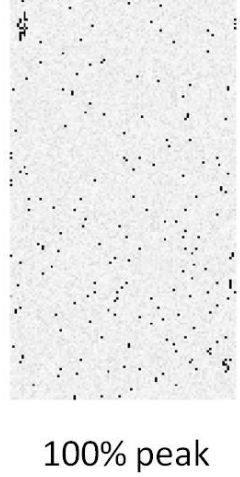

(d)

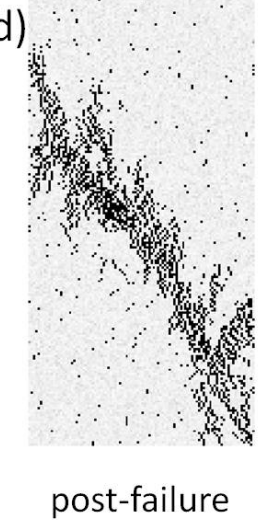

Figure 2: Evolution of microscopic failures. Micro fracturing initiate, propagate, interact each other. Finally, macroscopic failure plane across the specimen is formed. 


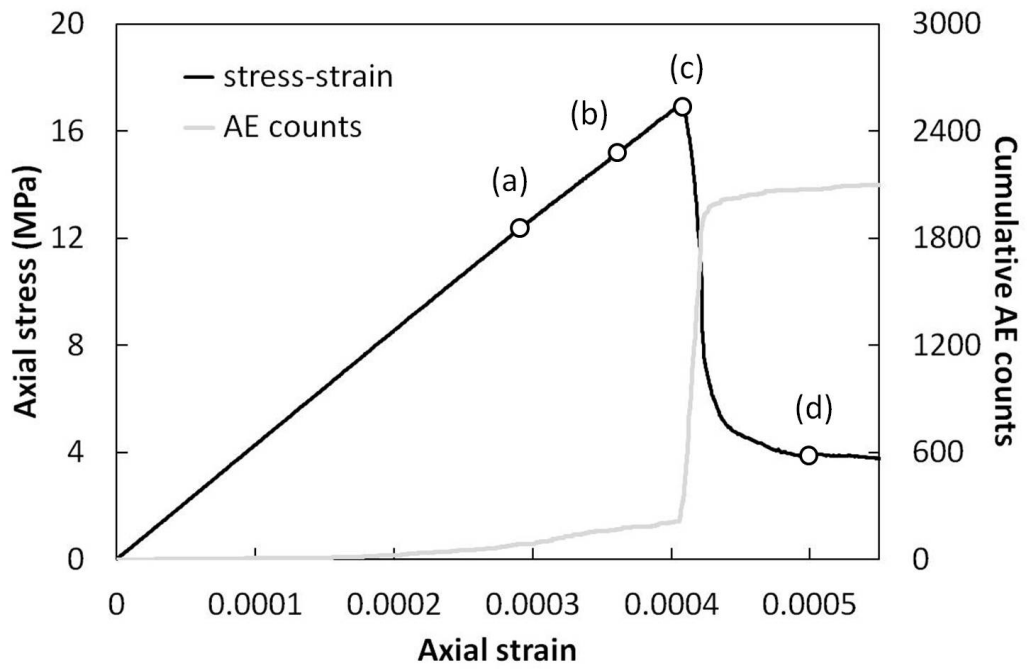

Figure 3: Stress-strain and cumulative AE counts-strain curves. Points (a)-(d) correspond with $75 \%, 90 \%$, $100 \%$ and post failure, respectively.

(2) $\mathrm{e}=48 \mathrm{~mm}, \mathrm{a}=14 \mathrm{~mm}$.

Figure 5 shows the failure patterns for case (1). We also show the laboratory test result ${ }^{11)}$. The macroscopic failure plane intersects with the hole. Figure 6 shows the failure patterns for case (2). The macroscopic failure plane is formed along the loading axis without intersecting with the hole. Our numerical results have good agreement with the laboratory experiments.

\section{CONCLUSIONS}

We developed the new approach for simulating the brittle failure of rock, especially from the viewpoint of the microscopic and macroscopic failure pattern. We, first, conducted uniaxial compressive test for validating the effectiveness of our approach. The results of our numerical simulation had good agreement with those from the previous studies. Next, we simulated the brittle failure of the disc with an eccentric hole of two different radii and eccentricities. The macroscopic failure pattern had good agreement with those from laboratory experiment. We conclude that the proposed approach can provide reasonable brittle failure of rock including complex free surfaces.

\section{REFERENCES}

1) C.A. Tang, H. Liu, P.K.K. Lee, Y. Tsui, and L.G. Tham, 2000, Numerical studies of the influence of microstructure on rock failure in uniaxial compression Part I: effect of heterogeneity, Int. J. Rock Mech. Min. Sci., 37, 555-569.

2) Z. Fang, and J.P. Harrison, 2002, Application of a local degradation model to the analysis of brittle fracture of laboratory scale rock specimens under triaxial conditions, Int. J. Rock Mech. Min. Sci., 39, 459-476.
3) Potyondy, D.O., and P.A. Cundall, 2004, A bonded-particle model for rock, Int. J. Rock Mech. Min. Sci., 41, 1329-1364.

4) Feng, X. T., P. Z., Pan, and H. Zhou, 2006, Simulation of the rock microfracturing process under uniaxial compression using an elasto-plastic cellular automaton, Int. J. Rock Mech. Min. Sci., 43, 1091-1108.

5) Ma, G.W., X.J., Wang, F. Ren, 2011, Numerical simulation of compressive failure of heterogeneous rock-like materials using SPH method, Int. J. Rock Mech. Min. Sci., 48, 353-363.

6) Suzuki, Y., Koshizuka, S., and Oka, Y., 2007, Hamiltonian moving-particle semi-implicit (HMPS) method for incompressible fluid flows, Comput. Methods Appl. Mech. Engrg., 196, 2876-2894.

7) Suzuki, Y., and Koshizuka, S., 2008, A Hamiltonian particle method for non-linear elastodynamics, Int. J. Numer. Meth. Engng., 74, 1344-1373.

8) Takekawa, J., Madariaga, R., Mikada, H., and Goto, T., 2012, Numerical simulation of seismic wave propagation produced by earthquake by using a particle method, Geophys. J. Int., 191, 1305-1316.

9) Takekawa, J., Mikada, H., and Goto, T., 2014, Numerical simulation using a Hamiltonian particle method for effective elastic properties in cracked media, Explor. Geophys., in press. doi: 10.1071/EG13098

10) Takekawa, J., Mikada, H., and Goto, T., 2014, A Hamiltonian particle method with a staggered particle technique for simulating seismic wave propagation, Pure Appl. Geophys., in press. doi: 10.1007/s00024-013-0763-x

11) Van de Steen, B., Vervoort, A., and Napier, J. A. L., 2005, Observed and simulated fracture pattern in diametrically loaded discs of rock material, Int. J. Frac., 131, 35-52. 


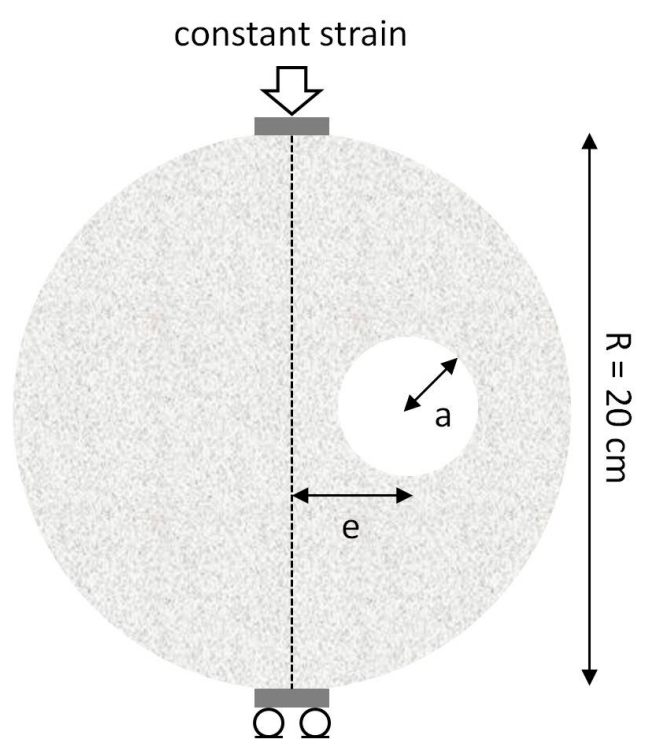

Figure 4: Configuration of a rock disc with an eccentric hole. "a" and "e" mean eccentricity and radius of the hole.
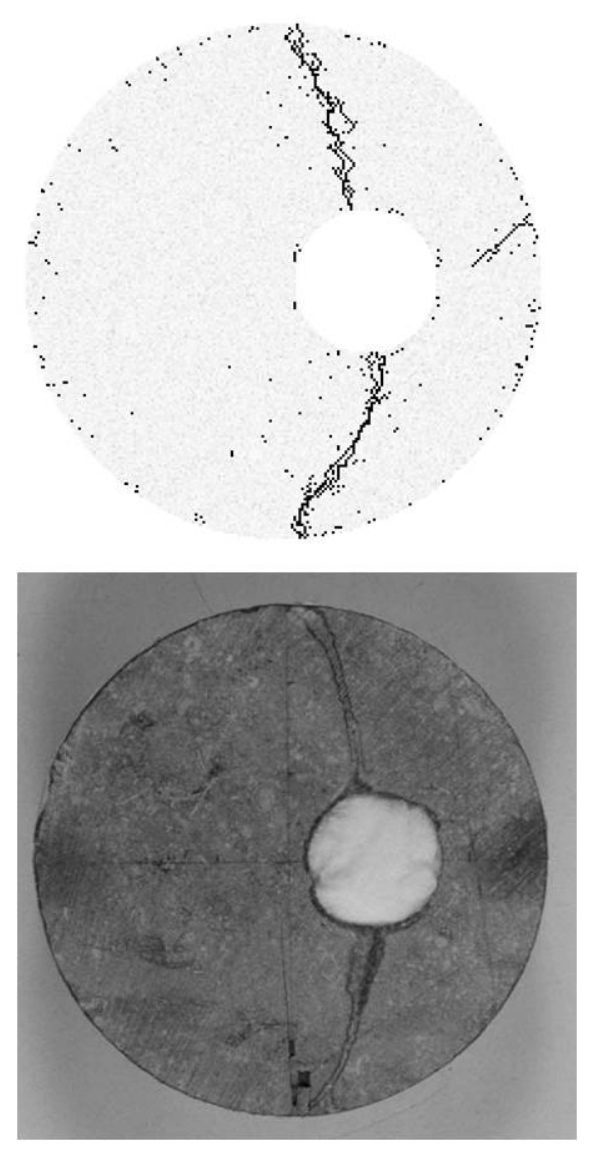

Figure 5: Failure pattern of case (1). Upper one is a numerical result. Lower one is an experimental result conducted by Van de Steen et al. (2005) ${ }^{11)}$.
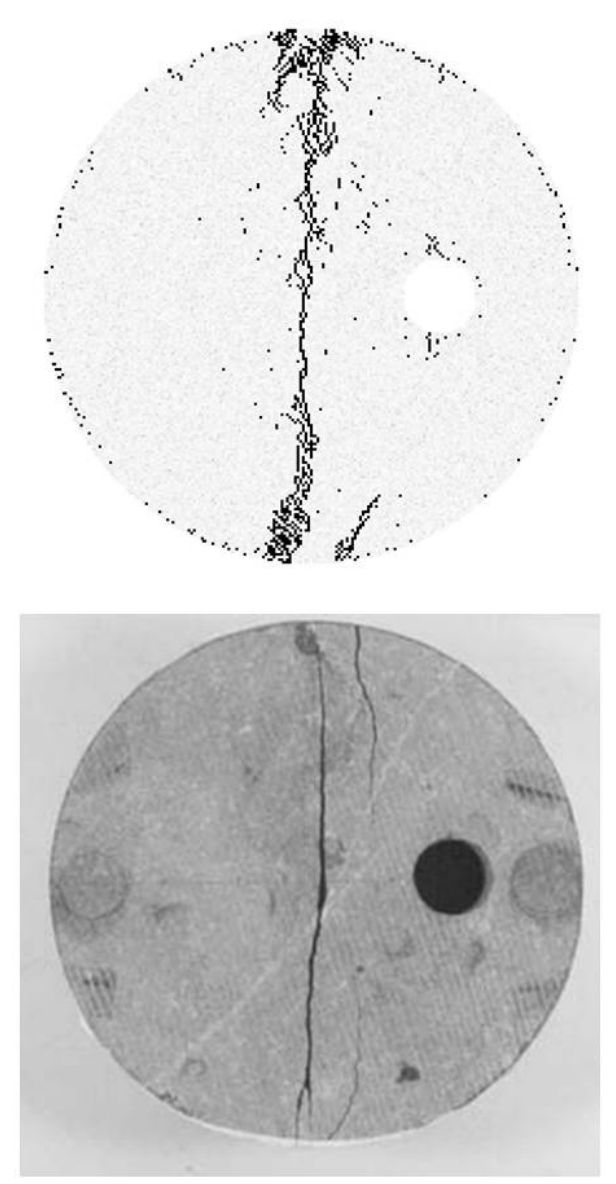

Figure 6: Failure pattern of case (2). Details are same as Figure 5. 